\title{
Embedding factorizations for 3-uniform hypergraphs II: $r$-factorizations into $s$-factorizations
}

\author{
Amin Bahmanian \\ Department of Mathematics \\ Illinois State University \\ Normal, Illinois, U.S.A.
}

\author{
Mike Newman * \\ Department of Mathematics and Statistics \\ University of Ottawa \\ Ottawa, Ontario, Canada \\ mnewman@uottawa.ca
}

Submitted: Nov 17, 2015; Accepted: May 18, 2016; Published: May 27, 2016

Mathematics Subject Classifications: 05C70, 05C65, 05C15, 05B40, 05B05

\begin{abstract}
Motivated by a 40-year-old problem due to Peter Cameron on extending partial parallelisms, we provide necessary and sufficient conditions under which one can extend an $r$-factorization of a complete 3 -uniform hypergraph on $m$ vertices, $K_{m}^{3}$, to an $s$-factorization of $K_{n}^{3}$. This generalizes an existing result of Baranyai and Brouwer-where they proved it for the case $r=s=1$.
\end{abstract}

Keywords: factorizations; embedding; detachments; amalgamations; hypergraphs; edge-colorings

\section{Introduction}

Let $V$ be a given finite set of cardinality $n$; the elements of $V$ will be called points. We denote the set of all $h$-subsets of $V$ by $\left(\begin{array}{l}V \\ h\end{array}\right)$. A parallelism of $\left(\begin{array}{l}V \\ h\end{array}\right)$ is a partition of $\left(\begin{array}{l}V \\ h\end{array}\right)$ whose classes are themselves partitions of $V$; the classes are called parallel classes. Note that a parallelism satisfies the usual Euclidean axiom for parallels: for every point $v \in V$ and for each $h$-subset $U$ of $V$, there is exactly one $h$-subset $U^{\prime}$ which is parallel to $U$ (that is, contained in the same parallel class as $U$ ) and contains $V$. Obviously, a parallelism can exist only if $h$ is a divisor of $n$. It was conjectured by Sylvester that this condition is sufficient as well, and Baranyai proved this conjecture [5]. The direction of research in similar subjects such as Steiner triple systems and Latin squares for which general existence theorems have been proved suggests the following problem.

Question 1. (Cameron [7, Question 1.2]) Under what conditions can partial parallelisms be extended to parallelisms?

\footnotetext{
*Research supported by NSERC
} 
There are different possible interpretations based on the precise notion of "partial" and "extend". To formulate this problem precisely, let us first introduce some basic terminology.

Let $K_{n}^{h}$ denote the complete $h$-uniform hypergraph on $n$ vertices which is a hypergraph on $n$ vertices whose edges are all $h$-subset of the vertex set. An $r$-factorization of a hypergraph is a partition (coloring) of the edges into $r$-regular spanning sub-hypergraphs. The following formulation of Problem 1 was investigated by the first author and Rodger in $[4]$ :

Question 2. Under what conditions can arbitrary edge-colorings of $K_{m}^{h}$ be extended to $r$-factorizations of $K_{n}^{h}$ ?

In this direction, it has been proven that

Theorem 3. (Bahmanian, Rodger [4, Theorem 3.1]) Suppose that $n \geqslant(2+\sqrt{2}) m$. Then a q-edge-coloring of $\mathcal{F}=K_{m}^{3}$ can be extended to an $r$-factorization of $K_{n}^{3}$ if and only if

(i) $3 \mid r n$,

(ii) $r \mid\left(\begin{array}{c}n-1 \\ 2\end{array}\right)$,

(iii) $q \leqslant\left(\begin{array}{c}n-1 \\ 2\end{array}\right) / r$, and

(iv) $d_{\mathcal{F}(j)}(v) \leqslant r$ for each $v \in V(\mathcal{F})$ and $1 \leqslant j \leqslant q$.

Here $d_{\mathcal{F}(j)}(v)$ is the degree of vertex $v$ in the sub-hypergraph of $\mathcal{F}$ induced by color $j$.

In this paper, we investigate the following formulation of Cameron's problem which is a special case of Problem 2: We are given $K_{n}^{h}$ which has $K_{m}^{h}$ as a sub-hypergraph, and the edges of $K_{m}^{h}$ have been colored so that the degree of each vertex within each color class is $r$ (so that we have an $r$-factorization of $K_{m}^{h}$ ). Can we color the remaining edges of $K_{n}^{h}$ so as to achieve an $s$-factorization of $K_{n}^{h}$ ?

Question 4. Under what conditions can an $r$-factorization of $K_{m}^{h}$ be extended to an $s$-factorization of $K_{n}^{h}$ ?

Baranyai and Brouwer [6] conjectured that a 1-factorization of $K_{m}^{h}$ can be extended to a 1-factorization of $K_{n}^{h}$ if and only if $h$ divides $m, n$, and $n \geqslant 2 m$. They proved this for $h=2,3$, and for arbitrary $h$ when $n$ is sufficiently large. This conjecture of Baranyai and Brouwer was beautifully settled by Häggkvist and Hellgren [9].

Theorem 5. (Häggkvist, Hellgren [9, Theorem 2]) Let $n=q t$ and $m=p t$, where $p \leqslant q / 2$. Suppose that we are given a coloring of a subgraph $K_{m}^{t}$, using $\left(\begin{array}{c}m-1 \\ t-1\end{array}\right)$ colors. Then this coloring can be extended to a coloring of $K_{n}^{t}$ using $\left(\begin{array}{c}n-1 \\ t-1\end{array}\right)$ colors.

In an attempt to generalize this result and extend Theorem 3 for larger values of $h$, in an earlier paper we showed that 
Theorem 6. (Bahmanian, Newman [3, Theorem 1.7]) If $\operatorname{gcd}(m, n, h)=\operatorname{gcd}(n, h)$, then an r-factorization of $K_{m}^{h}$ can be extended to an $r$-factorization of $K_{n}^{h}$ if and only if

(G1) $h|r m, h| r n$;

(G2) $r\left|\left(\begin{array}{c}m-1 \\ h-1\end{array}\right), r\right|\left(\begin{array}{c}n-1 \\ h-1\end{array}\right)$;

(G3) $n \geqslant 2 m$.

In this paper, we completely solve Problem 4 for $h=3$, which can be seen as an improvement of Theorem 3 for the case when the arbitrary edge-coloring of $K_{m}^{h}$ is replaced by a regular edge-coloring (see Theorem 16). Studying embedding factorization of graphs dates back to over 40 years ago, see for example the classical paper by Cruse [8], and its extensions by Andersen and Hilton [1]. For results concerning embedding connected factorization of graphs we refer the reader to $[10,11,12]$.

This paper is organized as follows. In Section 2, we discuss the necessary conditions. In Section 3, we give the prerequisites, and in Section 4, we prove our main result.

\section{General Necessary Conditions}

Throughout this paper we assume that $m, n, r, s, h \in \mathbb{N}$. Moreover, in order to avoid trivial cases we assume that

$$
h \geqslant 2 \text {, and } n>m>h .
$$

Lemma 7. If an r-factorization of $K_{m}^{h}$ can be embedded into an s-factorization of $K_{n}^{h}$, then

(N1) $h|r m, h| s n$;

(N2) $r\left|\left(\begin{array}{c}m-1 \\ h-1\end{array}\right), s\right|\left(\begin{array}{c}n-1 \\ h-1\end{array}\right)$;

(N3) $1 \leqslant s / r \leqslant\left(\begin{array}{c}n-1 \\ h-1\end{array}\right) /\left(\begin{array}{c}m-1 \\ h-1\end{array}\right)$;

(N4) $n \geqslant \frac{h}{h-1} m$ if $1<s / r<\left(\begin{array}{c}n-1 \\ h-1\end{array}\right) /\left(\begin{array}{c}m-1 \\ h-1\end{array}\right)$;

(N5) $n \geqslant 2 m$ if $s=r$.

Proof. Suppose that an $r$-factorization of $K_{m}^{h}$ can be embedded into an $s$-factorization of $K_{n}^{h}$. The degree sum of each $r$-factor in an $r$-factorization of $K_{m}^{h}$ is $r m$, which must be divisible by the size of each edge, $h$. On the other hand the degree of each vertex in $K_{m}^{h}$ is $\left(\begin{array}{c}m-1 \\ h-1\end{array}\right)$ which must be divisible by $r$. A similar argument shows that $h \mid s n$, and $s \mid\left(\begin{array}{c}n-1 \\ h-1\end{array}\right)$. This proves (N1) and (N2).

Let $q=\left(\begin{array}{c}m-1 \\ h-1\end{array}\right) / r, k=\left(\begin{array}{c}n-1 \\ h-1\end{array}\right) / s$. One can think of an $r$-factorization of $K_{m}^{h}$ as a $q$ edge-coloring in which each color class induces an $r$-factor. So we are extending a $q$-edgecoloring of $K_{m}^{h}$ to a $k$-edge-coloring of $K_{n}^{h}$ by extending each $r$-factor in $K_{m}^{h}$ to an $s$-factor in $K_{n}^{h}$, thus $s \geqslant r$ and $k \geqslant q$. In other words, $1 \leqslant s / r \leqslant\left(\begin{array}{c}n-1 \\ h-1\end{array}\right) /\left(\begin{array}{c}m-1 \\ h-1\end{array}\right)$. This proves (N3). 
For convenience, let us refer to the vertices in $V\left(K_{n}^{h}\right) \backslash V\left(K_{m}^{h}\right)$ as the new vertices, the edges in $E\left(K_{n}^{h}\right) \backslash E\left(K_{m}^{h}\right)$ as the new edges, and the colors in $\{q+1, \ldots, k\}$ as new colors if $k>q$.

Let $e_{j}$ be the number of edges of color $j$ in $K_{m}^{h}$ for $1 \leqslant j \leqslant k$. In an $s$-factorization of $K_{n}^{h}$, each of the $n-m$ new vertices is adjacent with exactly $s$ edges of each color class, therefore all the $n-m$ new vertices are adjacent with at most $s(n-m)$ edges of each color class. Since in an $s$-factorization of $K_{n}^{h}$ the number of hyperedges of each color class is $s n / h$, for $1 \leqslant j \leqslant k$ we have

$$
s(n-m)+e_{j} \geqslant s n / h .
$$

If $1<s / r<\left(\begin{array}{c}n-1 \\ h-1\end{array}\right) /\left(\begin{array}{c}m-1 \\ h-1\end{array}\right)$ (or $s>r$ and $k>q$ ), then since $e_{j}=0$ for $q+1 \leqslant j \leqslant k$, we have $s(n-m) \geqslant s n / h$ which proves (N4).

If $s / r=1$ (or $s=r$ ), fix a color $j \in\{1, \ldots, q\}$. Since $r=s$, there is no edge colored $j$ between $V\left(K_{m}^{h}\right)$ and the new vertices. Therefore, in order to to form an $s$-factor in $K_{n}^{h}$, there must be $r(n-m) / h$ edges colored $j$ in $K_{n-m}^{h}$ (the subgraph induced by the new vertices). But the total number of edges in $K_{n-m}^{h}$ is $\left(\begin{array}{c}n-m \\ h\end{array}\right)$. Therefore

$$
\left(\begin{array}{c}
n-m \\
h
\end{array}\right) \geqslant \frac{\left(\begin{array}{c}
m-1 \\
h-1
\end{array}\right)}{r} \frac{r(n-m)}{h} .
$$

Thus $\frac{h}{n-m}\left(\begin{array}{c}n-m \\ h\end{array}\right) \geqslant\left(\begin{array}{c}m-1 \\ h-1\end{array}\right)$ which implies $\left(\begin{array}{c}n-m-1 \\ h-1\end{array}\right) \geqslant\left(\begin{array}{c}m-1 \\ h-1\end{array}\right)$, and so $n-m-1 \geqslant m-1$, and so $n \geqslant 2 m$. This proves (N5) and the proof is complete.

Remark 8. Note that if $1=s / r=\left(\begin{array}{c}n-1 \\ h-1\end{array}\right) /\left(\begin{array}{c}m-1 \\ h-1\end{array}\right)$, then $n=m$ which is a trivial case.

\section{Fair Detachments of Hypergraphs}

If $x, y \in \mathbb{R}$, by $x \approx y$ we mean that $\lfloor y\rfloor \leqslant x \leqslant\lceil y\rceil$. For the purpose of this paper, a hypergraph $\mathcal{G}$ is a pair $(V(\mathcal{G}), E(\mathcal{G}))$ where $V(\mathcal{G})$ is a finite set called the vertex set, $E(\mathcal{G})$ is the edge multiset, where every edge is itself a multi-subset of $V(\mathcal{G})$. This means that not only can an edge occur multiple times in $E(\mathcal{G})$, but also each vertex can have multiple occurrences within an edge. By an edge of the form $\left\{u_{1}^{m_{1}}, u_{2}^{m_{2}}, \ldots, u_{r}^{m_{r}}\right\}$, we mean an edge in which vertex $u_{i}$ occurs $m_{i}$ times for $1 \leqslant i \leqslant r$. The total number of occurrences of a vertex $v$ among all edges of $E(\mathcal{G})$ is called the degree, $d_{\mathcal{G}}(v)$ of $v$ in $\mathcal{G}$. The multiplicity of an edge $e$ in $\mathcal{G}$, written $m_{\mathcal{G}}(e)$, is the number of repetitions of $e$ in $E(\mathcal{G})$ (note that $E(\mathcal{G})$ is a multiset, so an edge may appear multiple times). If $\left\{u_{1}^{m_{1}}, u_{2}^{m_{2}}, \ldots, u_{r}^{m_{r}}\right\}$ is an edge in $\mathcal{G}$, then we abbreviate $m_{\mathcal{G}}\left(\left\{u_{1}^{m_{1}}, u_{2}^{m_{2}}, \ldots, u_{r}^{m_{r}}\right\}\right)$ to $m_{\mathcal{G}}\left(u_{1}^{m_{1}}, u_{2}^{m_{2}}, \ldots, u_{r}^{m_{r}}\right)$. If $U_{1}, \ldots, U_{r}$ are multi-subsets of $V(\mathcal{G})$, then $m_{\mathcal{G}}\left(U_{1}, \ldots, U_{r}\right)$ means $m_{\mathcal{G}}\left(\bigcup_{i=1}^{r} U_{i}\right)$, where the union of $U_{i}$ s is the usual union of multisets. Whenever it is not ambiguous, we drop the subscripts; for example we write $d(v)$ and $m(e)$ instead of $d_{\mathcal{G}}(v)$ and $m_{\mathcal{G}}(e)$, respectively.

For a positive integer $h, \mathcal{G}$ is said to be $h$-uniform if $|e|=h$ for each $e \in E$. For a positive integer $r$, an $r$-factor in a hypergraph $\mathcal{G}$ is a spanning $r$-regular sub-hypergraph, and an $r$-factorization is a partition of the edge set of $\mathcal{G}$ into $r$-factors. The hypergraph 
$K_{n}^{h}:=\left(V,\left(\begin{array}{l}V \\ h\end{array}\right)\right)$ with $|V|=n$ (by $\left(\begin{array}{l}V \\ h\end{array}\right)$ we mean the collection of all $h$-subsets of $V$ ) is called a complete $h$-uniform hypergraph. A $k$-edge-coloring of $\mathcal{G}$ is a mapping $f: V(\mathcal{G}) \rightarrow C$ (often the set of colors $C$ is $\{1, \ldots, k\}$ ) and color class $j$ of $\mathcal{G}$, written $\mathcal{G}(j)$, is the subhypergraph of $\mathcal{G}$ induced by the edges of color $j$.

Let $\mathcal{G}$ be a hypergraph, let $U$ be some finite set, and let $\Psi: V(\mathcal{G}) \rightarrow U$ be a surjective mapping. The map $\Psi$ extends naturally to $E(\mathcal{G})$. For $A \in E(\mathcal{G})$ we define $\Psi(A)=\{\Psi(x)$ : $x \in A\}$. Note that $\Psi$ need not be injective, and $A$ may be a multiset. Then we define the hypergraph $\mathcal{F}$ by taking $V(\mathcal{F})=U$ and $E(\mathcal{F})=\{\Psi(A): A \in E(\mathcal{G})\}$. We say that $\mathcal{F}$ is an amalgamation of $\mathcal{G}$, and that $\mathcal{G}$ is a detachment of $\mathcal{F}$. Associated with $\Psi$ is a function $g$ defined by $g(u)=\left|\Psi^{-1}(u)\right|$; to be more specific we will say that $\mathcal{G}$ is a $g$-detachment of $\mathcal{F}$. Then $\mathcal{G}$ has $\sum_{u \in V(\mathcal{F})} g(u)$ vertices. Note that $\Psi$ induces a bijection between the edges of $\mathcal{F}$ and the edges of $\mathcal{G}$, and that this bijection preserves the size of an edge. We adopt the convention that it preserves the color also, so that if we amalgamate or detach an edge-colored hypergraph the amalgamation or detachment preserves the same coloring on the edges. We make explicit a straightforward observation: Given $\mathcal{G}, V(\mathcal{F})$ and $\Psi$ the amalgamation is uniquely determined, but given $\mathcal{F}, V(\mathcal{G})$ and $\Psi$ the detachment is in general far from uniquely determined.

We need the following special case of a general result in [2].

Theorem 9. (Bahmanian [2, Theorem 4.1]) Let $\mathcal{F}$ be a k-edge-colored hypergraph and let $g: V(\mathcal{F}) \rightarrow \mathbb{N}$. Then there exists a g-detachment $\mathcal{G}$ (possibly with multiple edges) of $\mathcal{F}$ whose edges are all sets, with amalgamation function $\Psi: V(\mathcal{G}) \rightarrow V(\mathcal{F}), g$ being the number function associated with $\Psi$, such that:

$$
d_{\mathcal{G}(j)}(v) \approx d_{\mathcal{F}(j)}(u) / g(u) \text { for each } u \in V(\mathcal{F}) \text {, each } v \in \Psi^{-1}(u) \text { and } 1 \leqslant j \leqslant k
$$

(F2) $m_{\mathcal{G}}\left(U_{1}, \ldots, U_{r}\right) \approx m_{\mathcal{F}}\left(u_{1}^{m_{1}}, \ldots, u_{r}^{m_{r}}\right) / \Pi_{i=1}^{r}\left(\begin{array}{c}g\left(u_{i}\right) \\ m_{i}\end{array}\right)$ for distinct $u_{1}, \ldots, u_{r} \in V(\mathcal{F})$ and $U_{i} \subset \Psi^{-1}\left(u_{i}\right)$ with $\left|U_{i}\right|=m_{i} \leqslant g\left(u_{i}\right)$ for $1 \leqslant i \leqslant r$.

An immediate consequence of Theorem 9 is the following that will be most useful throughout this paper.

Corollary 10. Let $\mathcal{F}$ be a hypergraph with vertex set $\{u, v\}$ such that $m\left(u^{i}, v^{h-i}\right)=$ $\left(\begin{array}{c}m \\ i\end{array}\right)\left(\begin{array}{c}n-m \\ h-i\end{array}\right)$ for $0 \leqslant i \leqslant h-1$. Then an $r$-factorization of $K_{m}^{h}$ can be embedded into an $s$-factorization of $K_{n}^{h}$ if and only if we can color the edges of $\mathcal{F}$ with $k$ colors so that

$$
\begin{aligned}
d_{j}(u)= & \begin{cases}m(s-r) & \text { for } 1 \leqslant j \leqslant q, \\
s m & \text { for } q+1 \leqslant j \leqslant k, \text { if } k>q,\end{cases} \\
& d_{j}(v)=s(n-m) \quad \text { for } 1 \leqslant j \leqslant k .
\end{aligned}
$$

where $q=\left(\begin{array}{c}m-1 \\ h-1\end{array}\right) / r, k=\left(\begin{array}{c}n-1 \\ h-1\end{array}\right) / s$, and $q, k \in \mathbb{N}$.

Proof. First, suppose an $r$-factorization of $K_{m}^{h}$ can be embedded into an $s$-factorization of $K_{n}^{h}$. By Lemma 7, $q, k$ both are integers. By removing the edges of $K_{m}^{h}$ from $K_{n}^{h}$, amalgamating those $m$ vertices in $K_{n}^{h}$ that belong to $K_{m}^{h}$ into a single vertex $u$, and the 
remaining $n-m$ vertices of $K_{n}^{h}$ into a vertex $v$, we obtain the hypergraph $\mathcal{F}$. The $k$-edgecoloring of $K_{n}^{h}$ (in which each color class is an $s$-factor) induces a $k$-edge-coloring in $\mathcal{F}$ that satisfies (2) and (3).

Conversely, suppose that an $r$-factorization of $K_{m}^{h}$ is given, and the edges of $\mathcal{F}$ are colored with $k$ colors so that (2) and (3) are satisfied. We show that we can embed the given $r$-factorization of $K_{m}^{h}$ into as $s$-factorization of $K_{n}^{h}$. Let $g: V(\mathcal{F}) \rightarrow \mathbb{N}$ with $g(u)=m, g(v)=n-m$. By Theorem 9, there exists a $g$-detachment $\mathcal{G}$ of $\mathcal{F}$ such that:

(a) By (F1), for each $w \in \Psi^{-1}(u)$

$$
d_{\mathcal{G}(j)}(w) \approx d_{j}(u) / g(u)= \begin{cases}m(s-r) / m=s-r & \text { for } 1 \leqslant j \leqslant q, \\ s m / m=s & \text { for } q+1 \leqslant j \leqslant k, \text { if } k>q,\end{cases}
$$

and for each $w \in \Psi^{-1}(v)$,

$$
d_{\mathcal{G}(j)}(w) \approx d_{j}(v) / g(v)=s(n-m) /(n-m)=s \text { for } 1 \leqslant j \leqslant k .
$$

(b) By $(\mathrm{F} 2), m_{\mathcal{G}}(U, V) \approx \frac{m\left(u^{i}, v^{h-i}\right)}{\left(\begin{array}{c}g(u) \\ i\end{array}\right)\left(\begin{array}{c}g(v) \\ h-i\end{array}\right)}=\frac{\left(\begin{array}{c}m \\ i\end{array}\right)\left(\begin{array}{c}n-m \\ h-i\end{array}\right)}{\left(\begin{array}{c}m \\ i\end{array}\right)\left(\begin{array}{c}n-m \\ h-i\end{array}\right)}=1$ for $U \subset \Psi^{-1}(u), V \subset \Psi^{-1}(v)$ with $|U|=i,|V|=h-i$, for $0 \leqslant i \leqslant h-1$.

Let us assume that $V\left(K_{m}^{h}\right)=\Psi^{-1}(u)$, and think of the given $r$-factorization of $K_{m}^{h}$ as a $q$-edge-coloring of $K_{m}^{h}$ so that each color class induces an $r$-factor. Let $\mathcal{H}$ be a hypergraph whose vertex set is $V(\mathcal{G})$, whose edges are $E\left(K_{m}^{h}\right) \cup E(\mathcal{G})$, and its edges are colored according to the colors of edges of $K_{m}^{h}$ and $\mathcal{G}$. Obviously, $\mathcal{H}$ contains an $r$-factorization of $K_{m}^{h}$. Moreover, the definition of $\mathcal{H}$ together with $(a)$ and (b) respectively implies that $d_{\mathcal{H}(j)}(x)=s$ for $1 \leqslant j \leqslant k$, and $\mathcal{H} \cong K_{n}^{h}$. This completes the proof.

\section{The Main Result}

In order to prove our main result, let us first review the obvious necessary conditions.

Lemma 11. If an r-factorization of $K_{m}^{3}$ can be embedded into an s-factorization of $K_{n}^{3}$, then

(C1) $3|r m, 3| s n$;

(C2) $r\left|\left(\begin{array}{c}m-1 \\ 2\end{array}\right), s\right|\left(\begin{array}{c}n-1 \\ 2\end{array}\right)$;

(C3) $1 \leqslant s / r \leqslant\left(\begin{array}{c}n-1 \\ 2\end{array}\right) /\left(\begin{array}{c}m-1 \\ 2\end{array}\right)$;

(C4) $n \geqslant 3 m / 2$ if $1<s / r<\left(\begin{array}{c}n-1 \\ 2\end{array}\right) /\left(\begin{array}{c}m-1 \\ 2\end{array}\right)$;

(C5) $n \geqslant 2 m$ if $s=r$;

(C6) $s m\left(\begin{array}{c}n-m \\ 2\end{array}\right) \geqslant\left(\begin{array}{c}n-1 \\ 2\end{array}\right)$ if $m(s-r)$ is odd and $s / r=\left(\begin{array}{c}n-1 \\ 2\end{array}\right) /\left(\begin{array}{c}m-1 \\ 2\end{array}\right)$. 
Proof. Taking $h=3$ in Lemma 7 proves (C1)-(C5). To prove (C6), suppose $m(s-r)$ is odd and $s / r=\left(\begin{array}{c}n-1 \\ 2\end{array}\right) /\left(\begin{array}{c}m-1 \\ 2\end{array}\right)$. If by contrary, $m\left(\begin{array}{c}n-m \\ 2\end{array}\right)<\left(\begin{array}{c}n-1 \\ 2\end{array}\right) / s$, and if $\mathcal{F}$ is the hypergraph described in Corollary 10, then there exists a color $j$ for which $m_{j}\left(u, v^{2}\right)=0$. Therefore, $m(s-r)=d_{j}(u)=2 m_{j}\left(u^{2}, v\right)$, contradicting the fact that $m(s-r)$ is odd.

For the rest of this section, we assume that (C1)-(C6) are satisfied, and that

$$
q:=\left(\begin{array}{c}
m-1 \\
2
\end{array}\right) / r, k:=\left(\begin{array}{c}
n-1 \\
2
\end{array}\right) / s
$$

Remark 12. A similar argument shows that it is necessary that

$$
m\left(\begin{array}{c}
n-m \\
2
\end{array}\right) \geqslant \begin{cases}k & \text { if } m, s \text { are odd and } r \text { is even, } \\
q & \text { if } m, r \text { are odd and } s \text { is even, } \\
k-q & \text { if } m, r, s \text { are odd }\end{cases}
$$

However, in Lemma 15 we will show that in most cases, (C4) implies this general necessary condition.

In order to prove that $(\mathrm{C} 1)-(\mathrm{C} 6)$ are also sufficient for an $r$-factorization of $K_{m}^{3}$ to be embedded into an $s$-factorization of $K_{n}^{3}$, we need to prove a few elementary results.

\section{Lemma 13.}

(a) $m\left[\left(\begin{array}{c}n-1 \\ 2\end{array}\right)-\left(\begin{array}{c}m-1 \\ 2\end{array}\right)\right]=2(n-m)\left(\begin{array}{c}m \\ 2\end{array}\right)+m\left(\begin{array}{c}n-m \\ 2\end{array}\right)$

(b) $(n-m)\left(\begin{array}{c}m \\ 2\end{array}\right)=m\left(\begin{array}{c}n-1 \\ 2\end{array}\right)-\left(\begin{array}{l}n \\ 3\end{array}\right)-2\left(\begin{array}{c}m \\ 3\end{array}\right)+\left(\begin{array}{c}n-m \\ 3\end{array}\right)$

Proof. Let $\mathcal{F}$ be a hypergraph with vertex set $\{u, v\}$ such that $m\left(u^{i}, v^{3-i}\right)=\left(\begin{array}{c}m \\ i\end{array}\right)\left(\begin{array}{c}n-m \\ 3-i\end{array}\right)$ for $0 \leqslant i \leqslant 2$. Counting the degree of $u$ in two different ways proves (a). Using part (a), we have the following that proves (b).

$$
\begin{aligned}
m\left(\begin{array}{c}
n-1 \\
2
\end{array}\right)-\left(\begin{array}{c}
n \\
3
\end{array}\right)-2\left(\begin{array}{c}
m \\
3
\end{array}\right)+\left(\begin{array}{c}
n-m \\
3
\end{array}\right)= & m\left(\begin{array}{c}
m-1 \\
2
\end{array}\right)+2(n-m)\left(\begin{array}{c}
m \\
2
\end{array}\right)+m\left(\begin{array}{c}
n-m \\
2
\end{array}\right)-2\left(\begin{array}{c}
m \\
3
\end{array}\right) \\
& -\left[\left(\begin{array}{c}
m \\
3
\end{array}\right)+\left(\begin{array}{c}
n-m \\
3
\end{array}\right)+(n-m)\left(\begin{array}{c}
m \\
2
\end{array}\right)+m\left(\begin{array}{c}
n-m \\
2
\end{array}\right)\right] \\
& -2\left(\begin{array}{c}
m \\
3
\end{array}\right)+\left(\begin{array}{c}
n-m \\
3
\end{array}\right) \\
= & (n-m)\left(\begin{array}{c}
m \\
2
\end{array}\right) .
\end{aligned}
$$

\section{Lemma 14.}

$$
(n-m)\left(\begin{array}{c}
m \\
2
\end{array}\right) \geqslant \begin{cases}q(s m-s n / 3-2 r m / 3)+(k-q)(s m-s n / 3) & \\
(k-q)(r m-r n / 3) & \text { if } r=s .\end{cases}
$$


Proof. To prove the first inequality, we have

$$
\begin{aligned}
q(s m-s n / 3-2 r m / 3)+(k-q)(s m-s n / 3) & =k(s m-s n / 3)-q(2 r m / 3) \\
& =(m-n / 3)\left(\begin{array}{c}
n-1 \\
2
\end{array}\right)-(2 m / 3)\left(\begin{array}{c}
m-1 \\
2
\end{array}\right) \\
& =m\left(\begin{array}{c}
n-1 \\
2
\end{array}\right)-\left(\begin{array}{c}
n \\
3
\end{array}\right)-2\left(\begin{array}{c}
m \\
3
\end{array}\right) \\
& <(n-m)\left(\begin{array}{c}
m \\
2
\end{array}\right),
\end{aligned}
$$

where the last inequality is true by Lemma 13(b).

If $r=s$, then by (C5) $n \geqslant 2 m$, and the following proves the second inequality.

$$
\begin{aligned}
(n-m)\left(\begin{array}{c}
m \\
2
\end{array}\right) & \geqslant(k-q)(r m-r n / 3) \\
& =(m-n / 3)\left[\left(\begin{array}{c}
n-1 \\
2
\end{array}\right)-\left(\begin{array}{c}
m-1 \\
2
\end{array}\right)\right] \Longleftrightarrow \\
3(n-m) m(m-1) & \geqslant(3 m-n)[(n-1)(n-2)-(m-1)(m-2)] \\
& =(3 m-n)(n-m)(n+m-3) \Longleftrightarrow \\
(n-m)[3 m(m-1)-(3 m-n)(n+m-3)] & \geqslant 0 \Longleftrightarrow \\
(n-3)(n-m)(n-2 m) & \geqslant 0 .
\end{aligned}
$$

Lemma 15.

$$
(n-m)\left(\begin{array}{c}
m \\
2
\end{array}\right) \leqslant q\lfloor m(s-r) / 2\rfloor+(k-q)\lfloor m s / 2\rfloor
$$

Proof. Let $\alpha=m(s-r) / 2-\lfloor m(s-r) / 2\rfloor, \beta=s m / 2-\lfloor s m / 2\rfloor$. Note that $\alpha, \beta \in\{0,1 / 2\}$, and

$$
\begin{aligned}
& 2 q\lfloor m(s-r) / 2\rfloor+2(k-q)\lfloor m s / 2\rfloor=\quad=\quad 2 q[m(s-r) / 2-\alpha]+2(k-q)(m s / 2-\beta) \\
& =k m s-q m r /-2 \alpha q-2 \beta(k-q) \\
& =m\left(\begin{array}{c}
n-1 \\
2
\end{array}\right)-m\left(\begin{array}{c}
m-1 \\
2
\end{array}\right)-2 \alpha q-2 \beta(k-q) \\
& \stackrel{\text { lem. } 13(a)}{=} 2(n-m)\left(\begin{array}{c}
m \\
2
\end{array}\right)+m\left(\begin{array}{c}
n-m \\
2
\end{array}\right)-2 \alpha q-2 \beta(k-q) \text {. }
\end{aligned}
$$

Therefore, (5) is equivalent to

$$
m\left(\begin{array}{c}
n-m \\
2
\end{array}\right) \geqslant 2 \alpha q+2 \beta(k-q) .
$$

If $k>q$, there are four cases to consider.

(a) $\alpha=\beta=0$ : In this case $m$ is even or $r, s$ are even, and so (6) is equivalent to $m\left(\begin{array}{c}n-m \\ 2\end{array}\right) \geqslant 0$ which is trivial. 
(b) $\alpha=0, \beta=1 / 2$ : In this case $m, s, r$ are odd, and so (6) is equivalent to $m\left(\begin{array}{c}n-m \\ 2\end{array}\right) \geqslant k-q$, and we have

$$
\begin{aligned}
m\left(\begin{array}{c}
n-m \\
2
\end{array}\right) & \geqslant \frac{\left(\begin{array}{c}
n-1 \\
2
\end{array}\right)}{s}-\frac{\left(\begin{array}{c}
m-1 \\
2
\end{array}\right)}{r} \Longleftrightarrow \\
r s m\left(\begin{array}{c}
n-m \\
2
\end{array}\right) & \geqslant r\left(\begin{array}{c}
n-1 \\
2
\end{array}\right)-s\left(\begin{array}{c}
m-1 \\
2
\end{array}\right) \\
& =r\left[\left(\begin{array}{c}
m-1 \\
2
\end{array}\right)+\left(\begin{array}{c}
n-m \\
2
\end{array}\right)+(n-m)(m-1)\right]-s\left(\begin{array}{c}
m-1 \\
2
\end{array}\right) \Longleftrightarrow \\
r(n-m)(m-1) & \leqslant r\left(\begin{array}{c}
n-m \\
2
\end{array}\right)(s m-1)+(s-r)\left(\begin{array}{c}
m-1 \\
2
\end{array}\right) .
\end{aligned}
$$

By (1) $m \geqslant 4$, but $m$ is odd, and so $m \geqslant 5$, which implies that $n-m \geqslant 3$. Therefore $\left(\begin{array}{c}n-m \\ 2\end{array}\right) \geqslant n-m$, which proves $(7)$.

(c) $\alpha=\beta=1 / 2$ : In this case $m, s$ are odd and $r$ is even, and so (6) is equivalent to $m\left(\begin{array}{c}n-m \\ 2\end{array}\right) \geqslant k$, and we have

$$
\begin{aligned}
s m\left(\begin{array}{c}
n-m \\
2
\end{array}\right) & \geqslant\left(\begin{array}{c}
n-1 \\
2
\end{array}\right) \\
& =\left(\begin{array}{c}
m-1 \\
2
\end{array}\right)+\left(\begin{array}{c}
n-m \\
2
\end{array}\right)+(n-m)(m-1) \quad \Longleftrightarrow \\
(s m-1)\left(\begin{array}{c}
n-m \\
2
\end{array}\right) & \geqslant\left(\begin{array}{c}
m-1 \\
2
\end{array}\right)+(n-m)(m-1) \\
(s m-1)(n-m)(n-m-1) & \geqslant(m-1)(m-2)+2(n-m)(m-1) \\
& =(m-1)(2 n-m-2) .
\end{aligned}
$$

Since $m$ is odd, by (1) $m \geqslant 5$, and we have $m^{2}-4 m+1 \geqslant 0$ or $(m+1)(m-3) \geqslant 2(m-2)$. But $m$ is odd and so by (C4) $n-m \geqslant \frac{m+1}{2}$ which implies $2(n-m)(n-m-2) \geqslant$ $\frac{m+1}{2}\left(\frac{m+1}{2}-2\right) \geqslant m-2$ and so $2(n-m)^{2}-4(n-m) \geqslant m-2$. Thus,

$$
2(n-m)(n-m-1)=2(n-m)^{2}-2(n-m) \geqslant 2 n-m-2 .
$$

Since $r$ is even, and $s$ is odd, we have $s>r \geqslant 2$. Therefore

$$
(s m-1)(n-m)(n-m-1)>2(m-1)(n-m)(n-m-1) \geqslant(m-1)(2 n-m-2) .
$$

(d) $\alpha=1 / 2, \beta=0$ : In this case $m, r$ are odd and $s$ is even, and thus (6) is equivalent to $m\left(\begin{array}{c}n-m \\ 2\end{array}\right) \geqslant q$. So we need to show that $r m\left(\begin{array}{c}n-m \\ 2\end{array}\right) \geqslant\left(\begin{array}{c}m-1 \\ 2\end{array}\right)$ or equivalently, $r m(n-$ $m)(n-m-1) \geqslant(m-1)(m-2)$. Since $m^{2}-4 m+7 \geqslant 0$, we have $(m+1)(m-1) \geqslant 4 m-8$, so $\frac{m+1}{2}\left(\frac{m+1}{2}-1\right) \geqslant m-2$, and since $r \geqslant 1$ and for $m$ odd by (C4), $n \geqslant m+\frac{m+1}{2}$, we have

$$
\begin{aligned}
r m(n-m)(n-m-1) & >(m-1)(n-m)(n-m-1) \\
& \geqslant(m-1) \frac{m+1}{2}\left(\frac{m+1}{2}-1\right) \geqslant(m-1)(m-2) .
\end{aligned}
$$


If $k=q$, there are two cases to consider.

(a) If $m(s-r)$ is even, then (6) is equivalent to $m\left(\begin{array}{c}n-m \\ 2\end{array}\right) \geqslant 0$ which is trivial.

(b) If $m(s-r)$ is odd, then (6) is equivalent to $m\left(\begin{array}{c}n-m \\ 2\end{array}\right) \geqslant q$ which is true by (C6).

Case $r=s$ of the following result is proved using a different method by the authors in $[3]$.

Theorem 16. An r-factorization of $K_{m}^{3}$ can be embedded into an s-factorization of $K_{n}^{3}$ if and only if

(C1) $3|r m, 3| s n$;

(C2) $r\left|\left(\begin{array}{c}m-1 \\ 2\end{array}\right), s\right|\left(\begin{array}{c}n-1 \\ 2\end{array}\right)$;

(C3) $1 \leqslant s / r \leqslant\left(\begin{array}{c}n-1 \\ 2\end{array}\right) /\left(\begin{array}{c}m-1 \\ 2\end{array}\right)$;

(C4) $n \geqslant 3 m / 2$ if $1<s / r<\left(\begin{array}{c}n-1 \\ 2\end{array}\right) /\left(\begin{array}{c}m-1 \\ 2\end{array}\right)$;

(C5) $n \geqslant 2 m$ if $s=r$;

(C6) $s m\left(\begin{array}{c}n-m \\ 2\end{array}\right) \geqslant\left(\begin{array}{c}n-1 \\ 2\end{array}\right)$ if $m(s-r)$ is odd and $s / r=\left(\begin{array}{c}n-1 \\ 2\end{array}\right) /\left(\begin{array}{c}m-1 \\ 2\end{array}\right)$.

Proof. The necessity is obvious by Lemma 11. To prove the sufficiency, let $\mathcal{F}$ be a hypergraph with vertex set $\{u, v\}$ such that $m\left(u^{i}, v^{3-i}\right)=\left(\begin{array}{c}m \\ i\end{array}\right)\left(\begin{array}{c}n-m \\ 3-i\end{array}\right)$ for $i=0,1,2$. By Corollary 10, it is enough to find a $k$-edge-coloring of $\mathcal{F}$ such that (2) and (3) are satisfied. In what follows, we find such a coloring. Observe that in any $k$-edge-coloring of $\mathcal{F}$, for $1 \leqslant j \leqslant k$ we have

$$
\begin{aligned}
& d_{j}(u)=2 m_{j}\left(u^{2}, v\right)+m_{j}\left(u, v^{2}\right), \text { and } \\
& d_{j}(v)=2 m_{j}\left(u, v^{2}\right)+m_{j}\left(u^{2}, v\right)+3 m_{j}\left(v^{3}\right) .
\end{aligned}
$$

There are two cases to consider.

Case 1. $s>\boldsymbol{r}$ : We color the edges of the form $\left\{u^{2}, v\right\}$ so that

$$
\begin{aligned}
& s m-s n / 3-2 r m / 3 \leqslant m_{j}\left(u^{2}, v\right) \leqslant m(s-r) / 2 \\
& s m-s n / 3 \leqslant m_{j}\left(u^{2}, v\right) \leqslant m s / 2 \quad \text { for } 1 \leqslant j \leqslant q, \\
& \text { for } q+1 \leqslant j \leqslant k, \text { if } k>q .
\end{aligned}
$$

In order to show that such a coloring is possible, first note that $m s / 2 \geqslant s m-s n / 3$ is equivalent to $n \geqslant 3 m / 2$, which is true if $k>q$ (by (C4)). Moreover, $m(s-r) / 2 \geqslant$ $s m-s n / 3-2 r m / 3$ is equivalent to $n \geqslant \frac{m}{2}(3-r / s)$ which is true by (1), and the following sequence of equivalences.

$$
\begin{aligned}
n \geqslant \frac{m}{2}(3-r / s)=\frac{m}{2}\left[3-\left(\begin{array}{c}
m-1 \\
2
\end{array}\right) /\left(\begin{array}{c}
n-1 \\
2
\end{array}\right)\right] & \Longleftrightarrow \\
2 n \geqslant 3 m-\frac{m(m-1)(m-2)}{(n-1)(n-2)} & \Longleftrightarrow \\
2 n(n-1)(n-2) \geqslant 3 m(n-1)(n-2)-m(m-1)(m-2) & \Longleftrightarrow \\
2(n-m)(n-m-1)(2 n+m-4) \geqslant 0 . &
\end{aligned}
$$


Therefore, it is enough to show that

$q(s m-s n / 3-2 r m / 3)+(k-q)(s m-s n / 3) \leqslant m\left(u^{2}, v\right) \leqslant q\lfloor m(s-r) / 2\rfloor+(k-q)\lfloor m s / 2\rfloor$,

which is true by Lemmas 14 and 15 .

Then, we color the edges of the form $\left\{u, v^{2}\right\}$ so that

$$
m_{j}\left(u, v^{2}\right)= \begin{cases}m(s-r)-2 m_{j}\left(u^{2}, v\right) & \text { for } 1 \leqslant j \leqslant q, \\ s m-2 m_{j}\left(u^{2}, v\right) & \text { for } q+1 \leqslant j \leqslant k, \text { if } k>q .\end{cases}
$$

This is possible, because by $(9) m_{j}\left(u, v^{2}\right) \geqslant 0$ for $1 \leqslant j \leqslant k$, and

$$
\begin{aligned}
& \sum_{j=1}^{k} m_{j}\left(u, v^{2}\right)=q m(s-r)+s m(k-q)-2 \sum_{j=1}^{k} m_{j}\left(u^{2}, v\right) \\
& =k s m-q r m-2(n-m)\left(\begin{array}{c}
m \\
2
\end{array}\right) \\
& =m\left(\begin{array}{c}
n-1 \\
2
\end{array}\right)-m\left(\begin{array}{c}
m-1 \\
2
\end{array}\right)-2(n-m)\left(\begin{array}{c}
m \\
2
\end{array}\right) \\
& \stackrel{\text { lem. }}{=}{ }^{3(a)} m\left(\begin{array}{c}
n-m \\
2
\end{array}\right)=m\left(u, v^{2}\right) \text {. }
\end{aligned}
$$

Finally, we color the edges of the form $\left\{v^{3}\right\}$ so that

$$
m_{j}\left(v^{3}\right)= \begin{cases}s n / 3-s m+m_{j}\left(u^{2}, v\right)+2 r m / 3 & \text { for } 1 \leqslant j \leqslant q, \\ s n / 3-s m+m_{j}\left(u^{2}, v\right) & \text { for } q+1 \leqslant j \leqslant k, \text { if } k>q .\end{cases}
$$

This coloring is possible, because by $(\mathrm{C} 1) m_{j}\left(v^{3}\right)$ is an integer for $1 \leqslant j \leqslant k$, by (9) $m_{j}\left(v^{3}\right) \geqslant 0$ for $1 \leqslant j \leqslant k$, and

$$
\begin{aligned}
\sum_{j=1}^{k} m_{j}\left(v^{3}\right) & =q(s n / 3-s m+2 r m / 3)+(k-q)(s n / 3-s m)+\sum_{j=1}^{k} m_{j}\left(u^{2}, v\right) \\
& =\quad(n-m)\left(\begin{array}{c}
m \\
2
\end{array}\right)+s k n / 3-s k m+2 q r m / 3 \\
& =(n-m)\left(\begin{array}{c}
m \\
2
\end{array}\right)+n\left(\begin{array}{c}
n-1 \\
2
\end{array}\right) / 3-m\left(\begin{array}{c}
n-1 \\
2
\end{array}\right)+2 m\left(\begin{array}{c}
m-1 \\
2
\end{array}\right) / 3 \\
& =(n-m)\left(\begin{array}{c}
m \\
2
\end{array}\right)+\left(\begin{array}{c}
n \\
3
\end{array}\right)-m\left(\begin{array}{c}
n-1 \\
2
\end{array}\right)+2\left(\begin{array}{c}
m \\
3
\end{array}\right) \\
& =\left(\begin{array}{c}
n-m \\
3
\end{array}\right)=m\left(v^{3}\right) .
\end{aligned}
$$

Using (8), we verify that the described edge-coloring satisfies (2) and (3).

$d_{j}(u)= \begin{cases}2 m_{j}\left(u^{2}, v\right)+m(s-r)-2 m_{j}\left(u^{2}, v\right)=m(s-r) & \text { for } 1 \leqslant j \leqslant q, \\ 2 m_{j}\left(u^{2}, v\right)+s m-2 m_{j}\left(u^{2}, v\right)=s m & \text { for } q+1 \leqslant j \leqslant k, \text { if } k>q .\end{cases}$ 
For $1 \leqslant j \leqslant q$,

$d_{j}(v)=3\left(s n / 3-s m+m_{j}\left(u^{2}, v\right)+2 r m / 3\right)+2\left(s m-r m-2 m_{j}\left(u^{2}, v\right)\right)+m_{j}\left(u^{2}, v\right)=s(n-m)$, and for $q+1 \leqslant j \leqslant k$, if $k>q$

$$
d_{j}(v)=3\left(s n / 3-s m+m_{j}\left(u^{2}, v\right)\right)+2\left(s m-2 m_{j}\left(u^{2}, v\right)\right)+m_{j}\left(u^{2}, v\right)=s(n-m) .
$$

Case 2. $\boldsymbol{r}=s$ : We color the edges of the form $\left\{u^{2}, v\right\}$ so that

$$
\begin{aligned}
& m_{j}\left(u^{2}, v\right)=0 \quad \text { for } 1 \leqslant j \leqslant q, \\
& r m-r n / 3 \leqslant m_{j}\left(u^{2}, v\right) \leqslant r m / 2 \text { for } q+1 \leqslant j \leqslant k .
\end{aligned}
$$

In order to show that such a coloring is possible, first note that $\mathrm{rm} / 2 \geqslant \mathrm{rm}-\mathrm{rn} / 3$ is equivalent to $n \geqslant 3 m / 2$, which is true by (C5). Therefore, it is enough to show that

$$
(k-q)(r m-r n / 3) \leqslant m\left(u^{2}, v\right) \leqslant(k-q)\lfloor r m / 2\rfloor,
$$

which is true by Lemmas 14 and 15 .

Then, we color the edges of the form $\left\{u, v^{2}\right\}$ so that

$$
m_{j}\left(u, v^{2}\right)= \begin{cases}0 & \text { for } 1 \leqslant j \leqslant q, \\ r m-2 m_{j}\left(u^{2}, v\right) & \text { for } q+1 \leqslant j \leqslant k .\end{cases}
$$

This is possible, because by $(10) m_{j}\left(u, v^{2}\right) \geqslant 0$ for $1 \leqslant j \leqslant k$, and

$$
\begin{aligned}
\sum_{j=1}^{k} m_{j}\left(u, v^{2}\right) & =\quad r m(k-q)-2 \sum_{j=q+1}^{k} m_{j}\left(u^{2}, v\right) \\
= & m\left(\begin{array}{c}
n-1 \\
2
\end{array}\right)-m\left(\begin{array}{c}
m-1 \\
2
\end{array}\right)-2(n-m)\left(\begin{array}{c}
m \\
2
\end{array}\right) \\
\stackrel{\text { lem. } 13(a)}{=} & m\left(\begin{array}{c}
n-m \\
2
\end{array}\right)=m\left(u, v^{2}\right) .
\end{aligned}
$$

Finally, we color the edges of the form $\left\{v^{3}\right\}$ so that

$$
m_{j}\left(v^{3}\right)= \begin{cases}r(n-m) / 3 & \text { for } 1 \leqslant j \leqslant q, \\ r n / 3-r m+m_{j}\left(u^{2}, v\right) & \text { for } q+1 \leqslant j \leqslant k .\end{cases}
$$

This coloring is possible, because by $(\mathrm{C} 1) m_{j}\left(v^{3}\right)$ is an integer for $1 \leqslant j \leqslant k$, by $(10)$ $m_{j}\left(v^{3}\right) \geqslant 0$ for $1 \leqslant j \leqslant k$, and

$$
\begin{aligned}
\sum_{j=1}^{k} m_{j}\left(v^{3}\right) & =\sum_{j=1}^{q} m_{j}\left(v^{3}\right)+\sum_{j=q+1}^{k} m_{j}\left(v^{3}\right) \\
& =\quad q r(n-m) / 3+(k-q)(r n / 3-r m)+m\left(u^{2}, v\right) \\
& =\quad(n-m)\left(\begin{array}{c}
m \\
2
\end{array}\right)+2 q r m / 3+k r n / 3-k r m \\
& =\quad(n-m)\left(\begin{array}{c}
m \\
2
\end{array}\right)+2\left(\begin{array}{c}
m \\
3
\end{array}\right)+\left(\begin{array}{c}
n \\
3
\end{array}\right)-m\left(\begin{array}{c}
n-1 \\
2
\end{array}\right) \\
& \stackrel{\text { lem. } 13(b)}{=}\left(\begin{array}{c}
n-m \\
3
\end{array}\right)=m\left(v^{3}\right) .
\end{aligned}
$$


Using (8), we verify that the described edge-coloring satisfies (2) and (3).

$$
d_{j}(u)= \begin{cases}0 & \text { for } 1 \leqslant j \leqslant q, \\ 2 m_{j}\left(u^{2}, v\right)+r m-2 m_{j}\left(u^{2}, v\right)=r m & \text { for } q+1 \leqslant j \leqslant k .\end{cases}
$$

For $1 \leqslant j \leqslant q$,

$$
d_{j}(v)=3 r(n-m) / 3=r(n-m)
$$

and for $q+1 \leqslant j \leqslant k$,

$$
d_{j}(v)=3\left(r n / 3-r m+m_{j}\left(u^{2}, v\right)\right)+2\left(r m-2 m_{j}\left(u^{2}, v\right)\right)+m_{j}\left(u^{2}, v\right)=r(n-m) .
$$

Applying Corollary 10 to $\mathcal{F}$, completes the proof.

\section{References}

[1] L. D. Andersen and A. J. W. Hilton. Generalized Latin rectangles. II. Embedding. Discrete Math., 31(3):235-260, 1980.

[2] M. A. Bahmanian. Detachments of hypergraphs I: The Berge-Johnson problem. Combin. Probab. Comput., 21(4):483-495, 2012.

[3] M. A. Bahmanian and Mike Newman. Extending factorizations of complete uniform hypergraphs. Submitted.

[4] M. A. Bahmanian and C. A. Rodger. Embedding factorizations for 3-uniform hypergraphs. J. Graph Theory, 73(2):216-224, 2013.

[5] Zs. Baranyai. On the factorization of the complete uniform hypergraph. In Infinite and finite sets (Colloq., Keszthely, 1973; dedicated to P. Erdös on his 60th birthday), Vol. I, pages 91-108. Colloq. Math. Soc. Jánōs Bolyai, Vol. 10. North-Holland, Amsterdam, 1975.

[6] Zs. Baranyai and A. E. Brouwer. Extension of colorings of the edges of a complete (uniform hyper)graph. Technical report, Mathematisch Centrum Amsterdam, Math. Centre Report ZW91, Zbl. 362.05059, 1977.

[7] Peter J. Cameron. Parallelisms of complete designs. Cambridge University Press, Cambridge-New York-Melbourne, 1976. London Mathematical Society Lecture Note Series, No. 23.

[8] Allan B. Cruse. On embedding incomplete symmetric Latin squares. J. Combin. Theory Ser. A, 16:18-22, 1974.

[9] R. Häggkvist and T. Hellgren. Extensions of edge-colourings in hypergraphs. I. In Combinatorics, Paul Erdös is eighty, Vol. 1, Bolyai Soc. Math. Stud., pages 215-238. János Bolyai Math. Soc., Budapest, 1993.

[10] A. J. W. Hilton, Matthew Johnson, C. A. Rodger, and E. B. Wantland. Amalgamations of connected $k$-factorizations. J. Combin. Theory Ser. B, 88(2):267-279, 2003. 
[11] Matthew Johnson. Amalgamations of factorizations of complete graphs. J. Combin. Theory Ser. B, 97(4):597-611, 2007.

[12] C. A. Rodger and E. B. Wantland. Embedding edge-colorings into 2-edge-connected $k$-factorizations of $K_{k n+1}$. J. Graph Theory, 19(2):169-185, 1995. 